\title{
Varied Properties of Hepatitis-Delta Virus-like Particles Produced by Baculovirus-Transduced Mammalian Cells
}

\author{
Ying-Wei Chiang ${ }^{\mathrm{a}}$, Jaw-Chin $\mathrm{Wu}^{\mathrm{b}, \mathrm{c}}$, Kuei-Chun Wang ${ }^{\mathrm{a}}$, Szu-Ting Chou ${ }^{\mathrm{a}}$ and $\mathrm{Yu}$-Chen $\mathrm{Hu}^{*}{ }^{* a}$ \\ ${ }^{a}$ Department of Chemical Engineering, National Tsing Hua University, Hsinchu 300, Taiwan \\ ${ }^{b}$ Institute of Clinical Medicine, National Yang Ming University, Taipei 112, Taiwan \\ ${ }^{c}$ Division of Gastroenterology, Department of Medicine, Taipei Veterans General Hospital, Taipei 112, Taiwan
}

\begin{abstract}
Hepatitis delta virus (HDV) is a defective virus that requires the supply of hepatitis B virus surface antigen (HBsAg) for replication and transmission. We have previously demonstrated that co-transduction of BHK cells with BacGD, a recombinant baculovirus expressing large hepatitis delta antigen (L-HDAg), and Bac-GS2, another recombinant baculovirus expressing HBsAg, gives rise to the assembly and secretion of $22 \mathrm{~nm}$ HBsAg subviral particles and 35-37 nm HDV-like particles (HDV VLP). In this study we uncovered oversize particles ( $>50 \mathrm{~nm}$ in diameter) comprised of $\mathrm{HBsAg}$ and L-HDAg and the particle properties varied with the relative dosages of Bac-GD and Bac-GS2, as demonstrated by Western blot, transmission electron microscopy and immunogold labeling. At a given Bac-GS2 dosage, decreasing the Bac-GD dosage resulted in the expression of more HBsAg, elevated secretion of HBsAg subviral particles, incorporation of more HBsAg into the HDV VLP, narrower particle size distribution and lower particle density. These data collectively demonstrated that the composition, and hence the properties, of HDV VLPs could be manipulated by altering the relative expression levels of structure proteins.
\end{abstract}

Keywords: Baculovirus, hepatitis delta virus, virus-like particle, L-HDAg, HBsAg.

\section{INTRODUCTION}

Hepatitis delta virus (HDV) is a satellite virus of hepatitis $B$ virus (HBV) and requires the supply of HBV surface antigen (HBsAg) for replication and transmission [1]. The HDV virion includes an outer envelope comprising lipids and HBsAg [2] and an inner ribonucleoprotein complex composed of a circular RNA genome as well as large and small forms of hepatitis delta antigens (L-HDAg and S-HDAg). HBsAg exists as large- (L), middle- (M) and small (S) forms in the HDV virion. These 3 isoforms are encoded in a single open reading frame and translated from different in-frame initiation codons, thus sharing a common C-terminal domain. $\mathrm{M}-\mathrm{HBsAg}$ carries a preS2 domain at the $\mathrm{N}$ terminus of S-HBsAg, while L-HBsAg carries a preS1 domain at the N terminus of M-HBsAg. Each of the three HBsAg exists in two forms with different degrees of glycosylation. The SHDAg and L-HDAg are identical in sequence except that LHDAg contains a C-terminal extension that allows for the isoprenylation of L-HDAg, which is crucial for HDV assembly and inhibition of HDV replication [3, 4].

VLPs (virus-like particles) are empty virus particles consisting of viral structural proteins and have captured growing interests as potential vaccine candidates $[5,6]$ because they can generally induce broad and strong immune responses [7]. It has been shown that co-expression of L-HDAg and SHBsAg leads to the assembly and secretion of 35-37 nm HDV-like particles (HDV VLP) [8,9]. In addition to the

*Address correspondence to this author at the Department of Chemical Engineering, National Tsing Hua University, Hsinchu 300, Taiwan; Tel: (886)3-571-8245; Fax: (886)3-571-5408; E-mail: yuchen@che.nthu.edu.tw
HDV VLP, $22 \mathrm{~nm}$ HBsAg subviral particles also arise from the over-expressed HBsAg [10].

Baculovirus (Autographa californica multiple nucleopolyhedrovirus, AcMNPV) has been widely employed for recombinant protein production in insect cells [11]. Since the finding that baculovirus is capable of transducing liver cells in 1995, the list of permissive cells, including primary and nondividing cells, has been steadily expanding (for review, see [11-13]). Thanks to the high transduction efficiency, non-replication nature, low cytotoxicity, large cloning capacity and ease of virus manipulation and production, baculovirus-mediated gene delivery has been exploited for in vivo gene therapy, viral vector production, study of gene functions (for review, see [11-13]), development of cell-based assays [14], surface display of eucaryotic proteins [15], cancer therapy [16], delivery of vaccine immunogens [17], modulation of chondrocyte differentiation [18], genetic modification of mesenchymal [19] and embryonic [20] stem cells, and bone tissue engineering [21].

Aside from these applications, we have previously constructed two recombinant baculoviruses: Bac-GD expressing L-HDAg $(27 \mathrm{kD})$ under the control of cytomegalovirus immediate-early (CMV-IE) promoter, and Bac-GS2 (previously designated Bac-GB) encoding the HBV PreS2/S/enhncer domains expressing HBsAg [22]. Transduction of mammalian cells with Bac-GS2 led to the expression of high levels of glycosylated $(27 \mathrm{kD})$ and unglycosylated $(24 \mathrm{kD}) \mathrm{S}-\mathrm{HBsAg}$ and relatively lower levels of M-HBsAg (33 kD). Cotransduction of mammalian cells (e.g. BHK and Huh-7) with Bac-GD and Bac-GS2 resulted in co-expression and efficient self-assembly and secretion of HDV VLP from the cells [22]. The production process was further transferred to a 
novel oscillating packed bed bioreactor, BelloCell, to mass produce the HDV VLPs [23].

Upon the optimization of HDV VLP production, intriguingly, we uncovered a heterogeneous particle size distribution with a minor fraction of particles as large as $\approx 70 \mathrm{~nm}$ (data now shown). Since HDV VLP was produced via the co-transduction with two baculoviruses, and the relative dosage of two recombinant baculoviruses affects the relative expression levels of structural proteins [23, 24], we suspected that the dosage of Bac-GD and Bac-GS2 influenced the VLP assembly, secretion and subsequent particle properties. To investigate this hypothesis, in this study we cotransduced the BHK cells with Bac-GD and Bac-GS2 using different dosages. The particles were collected from the medium, purified by $\mathrm{CsCl}$ gradient ultracentrifugation, and analyzed by Western blot/scanning densitometry, transmission electron microscopy (TEM) and immunogold labeling, so as to determine the protein secretion, particle density, size distribution and composition.

\section{MATERIALS AND METHODOLOGY}

\section{Cells and Medium}

Baby hamster kidney (BHK) cells were cultured in Dulbecco's minimal essential medium (DMEM, Sigma) supplemented with $10 \%$ heat inactivated fetal bovine serum (FBS; Gibco), 2 mM L-glutamine (Gibco) and non-essential amino acids (Gibco). The cells were maintained at $37^{\circ} \mathrm{C}$ in a humidified incubator at $5 \% \mathrm{CO}_{2}$. Insect cell Sf-9 was maintained in TNM-FH medium (Sigma) supplemented with $10 \%$ FBS at $27^{\circ} \mathrm{C}$ for baculovirus generation and amplification according to standard protocols [25]. The viruses were not concentrated by ultracentrifugation.

\section{Recombinant Baculoviruses}

The recombinant baculoviruses Bac-GD and Bac-GS2 (previously designated as Bac-GB) were constructed earlier [22]. Bac-GD harbored the cDNA encoding L-HDAg under the control of CMV-IE promoter. Bac-GS2 carried the gene sequences encoding the HBV PreS2/S and enhancer regions whereby the PreS2 region contained the endogenous promoter to drive the transcription of $\mathrm{M}$ and $\mathrm{S}$ forms of HBsAg. Both viruses also carried the egfp (enhanced green fluorescent protein) gene under the control of CMV-IE promoter as the reporter [22]. Because both Bac-GD and Bac-GS2 expressed EGFP in mammalian cells, which allowed for simple determination of transducing titers based on baculovirus' ability to transduce mammalian cells, herein the baculovirus transducing titers were measured according to a recently developed titration protocol [26] and are expressed as transducing units $(\mathrm{TU}) / \mathrm{ml}$. Correspondingly, the virus dosages per cell (TU/cell) are expressed as multiplicity of transduction (MOT) as described [27], rather than the common multiplicity of infection (MOI).

\section{Baculovirus Transduction}

A simple process eliminating the need for virus ultracentrifugation [22] was adopted for transduction. Briefly, the Bac-GD and Bac-GS2 virus solutions were mixed together based on the necessary virus dosage (MOT) intended to use. The volume of the transduction solution was adjusted with phosphate-buffered saline (PBS) to $8 \mathrm{ml}$ for each $15-\mathrm{cm}$ dish. Meanwhile, BHK cells were incubated in $15-\mathrm{cm}$ dishes $\left(2 \times 10^{7}\right.$ cells per dish) overnight and washed with PBS prior to transduction. Transduction was initiated by adding the transduction solution and continued by shaking the dishes on a rocking platform for $6 \mathrm{~h}$ at $25^{\circ} \mathrm{C}$. After the virus incubation period, the virus solution was aspirated and the cells were washed with PBS again. The wells were replenished with $20 \mathrm{ml}$ complete medium containing $2 \mathrm{mM}$ sodium butyrate and incubated at $37{ }^{\circ} \mathrm{C}$. The medium exchange was performed at 2 days post-transduction (dpt) and the culture was harvested at $4 \mathrm{dpt}$.

\section{Purification of VLPs by Ultracentrifugation}

The culture medium collected at 2 and $4 \mathrm{dpt}$ was centrifuged at $12,000 \mathrm{rpm}$ for $30 \mathrm{~min}$ to remove cellular debris. The medium was then concentrated by Stirred Cell ${ }^{\circledast}$ (Millipore) using a $300 \mathrm{kD}$ ultrafiltration disc. The VLPs in the retenate were pelleted by ultracentrifugation (P40ST rotor, Hitachi) through $20 \%$ sucrose in TE buffer (10 mM Tris, 1 mM EDTA, pH 8) at 38,000 rpm for $5 \mathrm{~h}$, and subsequently purified by $10-40 \% \mathrm{CsCl}$ gradient ultracentrifugation $(38,000 \mathrm{rpm}, 24 \mathrm{~h})$. The aliquots $(700 \mu \mathrm{l})$ of $\mathrm{CsCl}$ gradient were fractionated from bottom to top and analyzed by Western blot (see below) for the detection of HBsAg and LHDAg. The density of each fraction was determined by a refractometer. The HDV VLP-positive fractions containing both HBsAg and L-HDAg were pooled and ultracentrifuged $(38,000 \mathrm{rpm}, 5 \mathrm{~h})$ again. The pellets were resuspended in 50 $\mu \mathrm{l}$ deionized water for Western blot, transmission electron microscopy (TEM) and immunogold labeling.

\section{Western Blot}

To detect intracellular HBsAg and L-HDAg, the cells were harvested at $4 \mathrm{dpt}$ and lysed in lysis buffer (1\% NP-40, $1 \%$ TritonX-100, $0.5 \%$ sodium deoxycholate, $1 \mu \mathrm{g} / \mathrm{ml}$ aprotinin, $1 \mathrm{mM}$ phenylmethylsulfonylfluoride, $10 \mathrm{mM}$ Tris, $1 \mathrm{mM}$ EDTA, pH 7.4) on ice for $30 \mathrm{~min}$. To detect the secreted HBsAg and L-HDAg, the particles were purified as above. The intracellular or purified samples were resolved on $12 \%$ gels and transferred onto nitrocellulose membranes as described previously [22]. The L-HDAg was probed by human anti-HDV sera [28] while HBsAg was probed by A10F1 MAb [29]. The secondary antibodies were either goat AP- (anti-human) or HRP-conjugated (anti-mouse) IgG (Kirkegaard and Perry Laboratories). The membranes were developed with either BCIP/NBT color developing reagent (Sigma) or chemiluminescence reagent (Pierce).

For quantitative analysis of the VLP composition, known amounts of purified S-HDAg (kindly provided by Prof. PeiJer Chen) and HBsAg were serially diluted and included in the Western blot analysis. The membranes were scanned and analyzed by Scion Image (Scion Corporation).

\section{TEM and Immunogold Labeling}

The diameter and morphology of the purified particles were examined by TEM (Hitachi, H7500) as described previously [23]. Histograms were generated by analyzing at least 5 representative micrographs with an imaging software (Optimas, Optimas Corporation) to measure the particle 
number with a certain diameter. Each data represents the measurement of at least 300 particles.

For immunogold labeling, the purified particles were dripped onto copper grids and probed by mouse anti-HBsAg MAb (A10F1). The secondary antibody was goat anti-mouse IgG conjugated with $5 \mathrm{~nm}$ gold particles (Sigma). After washing of the unbound gold particles, the grids were stained by $2 \%$ phosphotungstic acid and observed by TEM.

\section{RESULTS}

\section{Effects of Baculovirus Dosage on VLP Density}

To explore whether the relative virus dosage of Bac-GD and Bac-GS2 affected the particle properties, we cotransduced the BHK cells with Bac-GD and Bac-GS2 at different multiplicities of transduction (MOT). The experimental design was simplified by fixing the Bac-GS2 MOT at 10 while varying the Bac-GD MOT to 20,5 or 2 . These virus dosages were sufficient to yield transduction efficiencies greater than $90 \%$. For simplicity, the Bac-GD MOT is denoted as MOT throughout the text.

To examine whether all the HDV VLPs assembled inside the cells were effectively secreted, the co-transduced cell lysates were subject to Western blot using anti-HBsAg and anti-HDAg antibodies. Fig. (1a) reveals an increasing amount of intracellular $27 \mathrm{kD}$ L-HDAg (which is nucleusbound in nature) with ascending Bac-GD MOT, suggesting an increasing degree of L-HDAg overexpression. In contrast, no HBsAg accumulated within the cells regardless of the MOT. Since HBsAg is incorporated into HBsAg subviral particles or HDV VLP [22], the data indicated that the majority, if not all, of the expressed HBsAg and hence all HDV VLPs, secreted out of the cells.

The particles secreted into the medium were harvested, concentrated by ultrafiltration and purified by $\mathrm{CsCl}$ gradient ultracentrifugation. The fractions after $\mathrm{CsCl}$ gradient ultracentrifugation were collected and analyzed by Western blot. Fig. (1b) depicts that for all three MOT the HDV VLPs comprised of HBsAg and L-HDAg secreted into the medium, but the major fractions characterized by the predominant co-existence of L-HDAg and two forms of HBsAg (MHBsAg and S-HBsAg) shifted from fraction 7 (for MOT 20) to fractions 8 (for MOT 5) and 9 (for MOT 2), respectively. The shift corresponded to changes in the density distribution from 1.24-1.29 $\mathrm{g} / \mathrm{cm}^{3}$ (fractions 6-8) for MOT 20 to 1.22$1.27 \mathrm{~g} / \mathrm{cm}^{3}$ (fractions 7-9) for MOT 5, and to 1.20-1.25 $\mathrm{g} / \mathrm{cm}^{3}$ (fractions 8-10) for MOT 2. Specifically, the major bands containing the most abundant HBsAg and L-HDAg sedimented at $1.27 \mathrm{~g} / \mathrm{cm}^{3}$ (MOT 20), $1.25 \mathrm{~g} / \mathrm{cm}^{3}$ (MOT 5) and $1.24 \mathrm{~g} / \mathrm{cm}^{3}$ (MOT 2), respectively. The particle densities were somewhat larger than that of the HDV VLP $(\approx 1.20$ $\mathrm{g} / \mathrm{cm}^{3}$ ) produced in yeasts [10]. These data collectively delineated a direct correlation between the particle density and the Bac-GD MOT.

\section{Effects of Baculovirus Dosage on VLP Size Distribution}

To explore how Bac-GD MOT affected the VLP size distribution, the HDV VLPs-positive fractions were pooled, pelleted by ultracentrifugation again and examined by TEM. For all three MOT (Fig. 2a,c), $22 \mathrm{~nm}$ particles resembling (a)

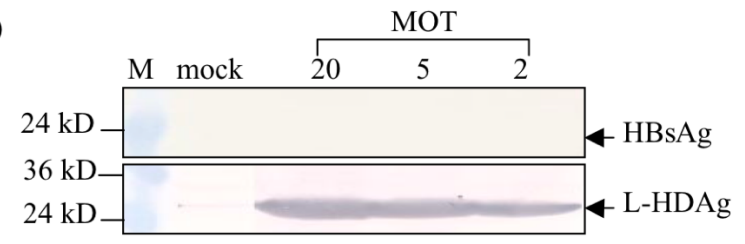

(b)

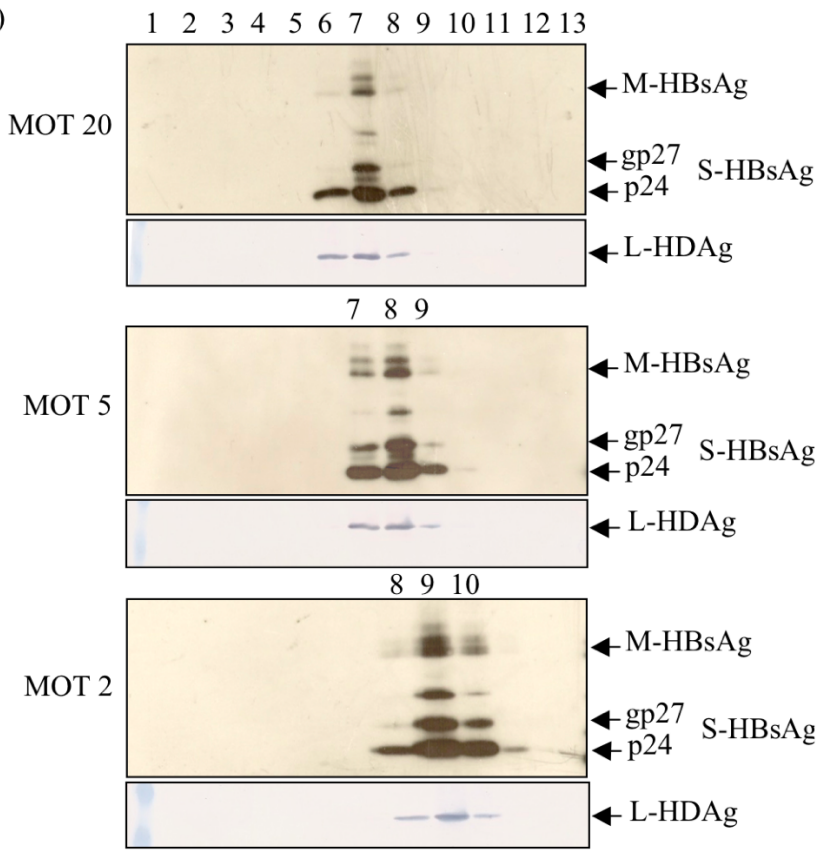

Fig. (1). Effects of Bac-GD MOT on the VLP density. (a) Analysis of cell lysates. (b) Analysis of purified particles. BHK cells $\left(2 \times 10^{7}\right.$ cells) were cultured in $15-\mathrm{cm}$ dishes and co-transduced with BacGD at varying MOT (20, 5 or 2) and Bac-GS2 at a fixed MOT 10. BHK cells are highly susceptible to baculovirus transduction [22] and the transduction efficiencies exceeded $90 \%$ at MOT 2 (data not shown). The medium and the cells were harvested at 4 days posttransduction (dpt). The medium was ultrafiltrated by Stirred Cell ${ }^{\circledR}$, and subjected to sucrose and $\mathrm{CsCl}$ ultracentrifiguation. The fractions were collected from bottom to top (700 $\mu \mathrm{l} /$ fraction) and analyzed by Western blot using anti-HBsAg and anti-HDAg Ab. The data are representative of 4 independent experiments. Mock represents mock transduction. M, marker.

the HBsAg subviral particles, $\approx 37 \mathrm{~nm}$ particles resembling the authentic HDV virions and other particles with heterogeneous size distributions were observed. Notably, MOT 20 resulted in a wider size distribution $(\approx 22-90 \mathrm{~nm}$, Fig. 2a) with a considerable fraction of oversize particles while MOT 5 led to a narrower size distribution with only a minor fraction of oversize particles (up to $\approx 60 \mathrm{~nm}$, Fig. 2b). Comparatively, MOT 2 yielded a more homogeneous size distribution whereby most of the particles had diameters of $\approx 22-37 \mathrm{~nm}$ (Fig. 2c) despite the presence of some oversize particles (up to $50 \mathrm{~nm}$, not shown). It should be noted that although some intracellular organelles are similar to these particles in size (e.g. endoplasmic reticulum $\approx 40-70 \mathrm{~nm}$, ribosomes $\approx 20 \mathrm{~nm}$ ), the VLPs were collected from the extracellular medium containing scarce cellular components (because baculovirus does not cause cell death and lysis) and the cell debris was removed prior to VLP purification, it was fairly unlikely that these oversize particles originated from cellular organelles. 

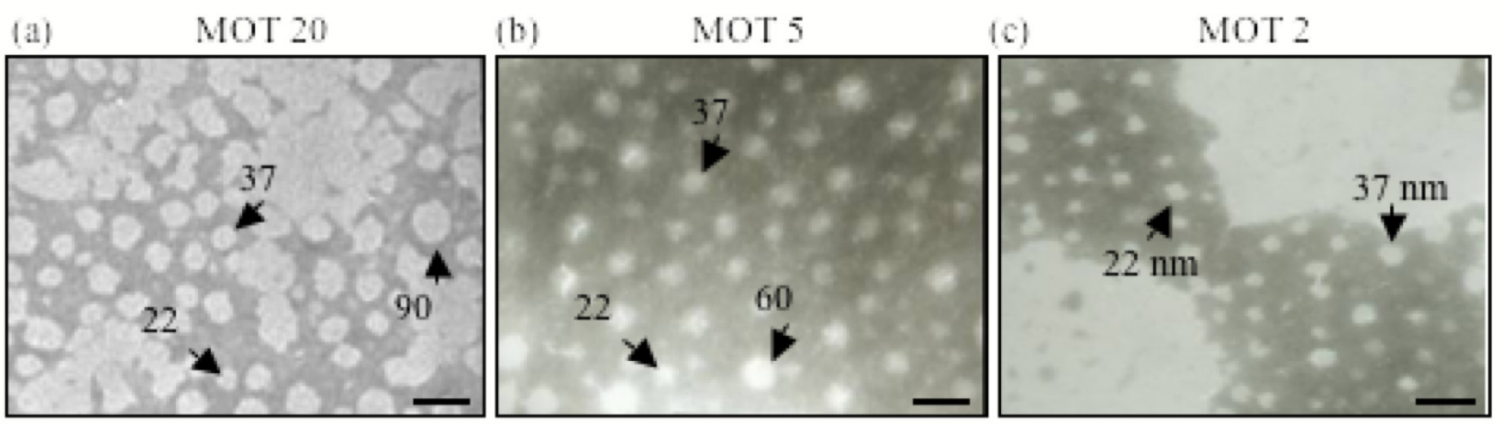

Fig. (2). Effects of MOT on VLP size distribution. The BHK cells were co-transduced with Bac-GS2 (MOT 10) and Bac-GD (MOT 20, 5 or 2) as described in Fig. (1). The medium harvested at $4 \mathrm{dpt}$ was ultrafiltrated by Stirred Cell ${ }^{\mathbb{R}}$, subjected to sucrose and CsCl ultracentrifiguation, and the fractions containing VLPs were pelleted again. The pellets were resuspended in $\mathrm{H}_{2} \mathrm{O}$ and then examined by TEM. The data are representative of 4 independent experiments. Bar, $100 \mathrm{~nm}$.

The purified particles were characterized by immunogold labeling to further confirm the identity (Fig. 3a,c). As expected, the oversize particles (e.g. the $90 \mathrm{~nm}$ particles in Fig. (3a), the $70 \mathrm{~nm}$ particle in Fig. 3b and the $50 \mathrm{~nm}$ particle in Fig. (3c)) also reacted with the anti-HBsAg antibody, indicating that they also consisted of HBsAg.

To correlate the MOT and particle size distribution, the electron micrographs were processed using an image processing software to count the number of particles with a certain size. The histogram (Fig. 4a) depicts that at MOT 20 the $>40 \mathrm{~nm}$ particles were predominant $(\approx 42.8 \%)$ in the particle population whereas the particles whose sizes matched those of the authentic HDV $(\approx 35-40 \mathrm{~nm})$ and HBsAg subviral particles $(\approx 20-25 \mathrm{~nm})$ accounted for $\approx 20.9 \%$ and $\approx 5.8 \%$, respectively. MOT 5 (Fig. $4 \mathbf{b}$ ) yielded a relatively uniform distribution of particles with various diameter ranges, except that the $20-25 \mathrm{~nm}$ subviral particles accounted for $\approx 10.1 \%$. Conversely, at MOT 2 the $>40 \mathrm{~nm}$ particles accounted for only $\approx 8.4 \%$, while the $35-40 \mathrm{~nm}$ and $20-25 \mathrm{~nm}$ particles accounted for $\approx 20.6 \%$ and $21.2 \%$, respectively.

\section{Effects of Baculovirus Dosage on VLP Composition}

To elucidate the dependency of particle composition on Bac-GD MOT, we quantified the amounts of HBsAg and LHDAg secreted to the medium by Western blot, in which known amounts of purified S-HDAg and HBsAg were serially diluted and used as the standards. The bands in the Western blots were scanned and the intensities quantified using Scion Image. As delineated in Table 1, the decrease in Bac-GD MOT from 20 to 2 resulted in an increase in HBsAg secretion from $\approx 31.3 \mathrm{ng}$ to $\approx 83.1 \mathrm{ng}$ per $10^{7}$ cells. Intriguingly, decreasing the MOT from 20 to 2 also elevated the LHDAg yields in the secreted particles from $\approx 39.4 \mathrm{ng}$ to $\approx 59.9$ ng per $10^{7}$ cells.

It is established that HBsAg overexpression leads to the self-assembly of subviral particles exclusively from the excess HBsAg. To quantify the composition of HDV VLPs derived from the interactions of L-HDAg and HBsAg while excluding the contributions from the HBsAg subviral particles, the 20-25 nm particles were defined as the HBsAg particles, while other particles with diameters exceeding $25 \mathrm{~nm}$ were defined as the HDV VLP. Therefore, the HBsAg subviral particles accounted for $\approx 5.8 \%, 10.1 \%$ and $21.2 \%$ of total particles at MOT 20,5 and 2, respectively (Fig. 4a,c and Table 1). Accordingly, the HDV VLP accounted for $\approx 94.2 \%$, $\approx 89.9 \%$ and $\approx 78.8 \%$ of total particles at MOT 20,5 and 2 , respectively (Table 1). The amounts of HBsAg associated with the HDV VLP were calculated by subtracting the amounts of HBsAg in the subviral particles from the total amounts of secreted HBsAg. Hence the weight ratios of L$\mathrm{HDAg} / \mathrm{HBsAg}$ (D/B ratio) were calculated to be 1.26 at MOT 20, 0.86 at MOT 5 and 0.72 at MOT 2 (Table 1). The declining $\mathrm{D} / \mathrm{B}$ ratio attested that the amounts of L-HDAg incorporated into the HDV VLP decreased with descending Bac-GD MOT. (a)

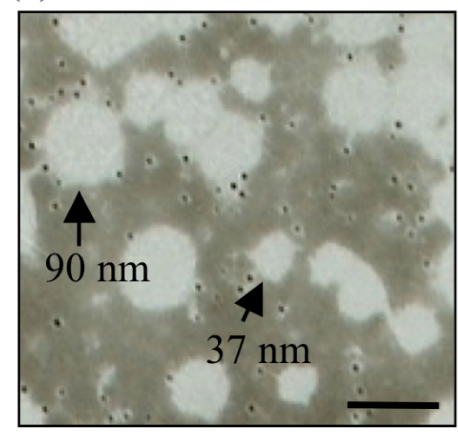

(b)

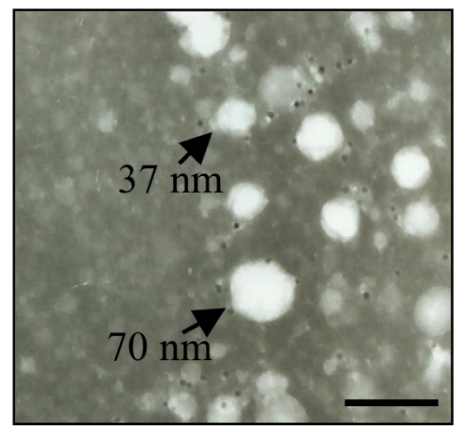

(c) MOT 2

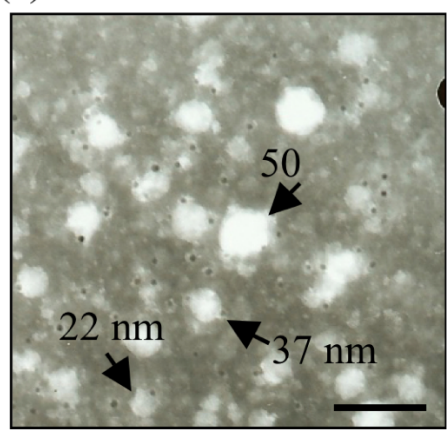

Fig. (3). Characterization of particles by immunogold labeling. The particles were produced using different Bac-GD MOT (20, 5 or 2) and purified as in Fig. (2). The particles were subjected to immunogold labeling using anti-HBsAg Ab as the primary antibody and goldconjugated anti-mouse $\mathrm{Ab}$ as the secondary $\mathrm{Ab}$. The data are representative of 4 independent experiments. Bar, $100 \mathrm{~nm}$. 
(a)

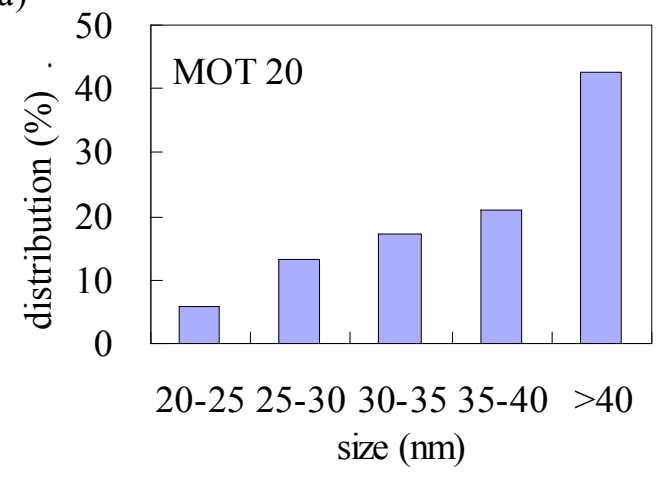

(b)

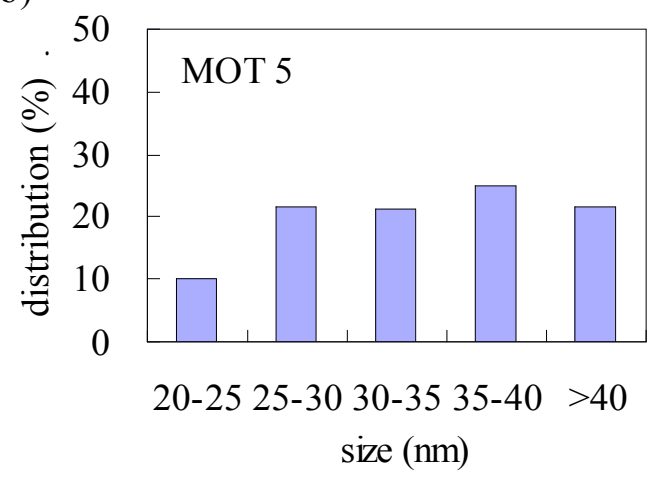

(c)

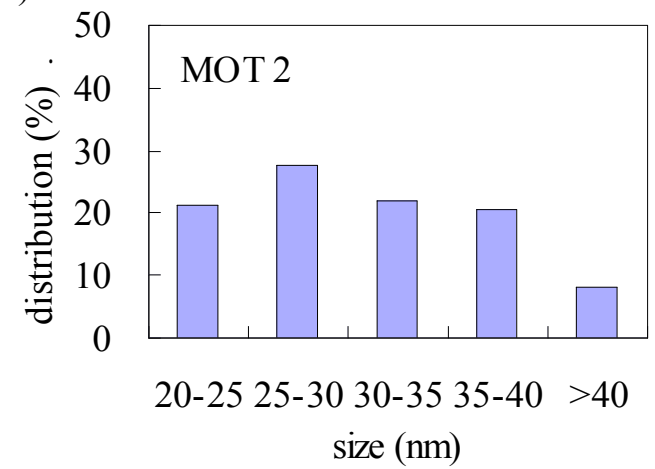

Fig. (4). Variation of VLP size distribution on Bac-GD MOT. (a) MOT 20; (b) MOT 5; (c) MOT 2. The TEM micrographs representative of 4 independent experiments were processed with an image processing software and the number of particles of certain sizes were counted. For each MOT, at least 300 particles were counted.

\section{DISCUSSION}

Herein we demonstrated that co-transduction of BHK cells with Bac-GD and Bac-GS2 led to co-expression of L-
HDAg and HBsAg, and ensuing secretion of HBsAg subviral particles and HDV VLPs. Interestingly, for a given BacGS2 dosage (MOT 10), decreasing the Bac-GD MOT from 20 to 2 considerably elevated the secretion levels of not only HBsAg but also L-HDAg (Table 1). The elevated secretion level at MOT 2 could be ascribed to the reduced L-HDAg expression level, which not only competed with HBsAg for cellular resources for expression but also suppressed the HBsAg expression [29]. L-HDAg is a nuclear protein that interacts with HBsAg for HDV VLP assembly and secretion, thus its excessive expression relative to HBsAg resulted in the accumulation of intracellular proteins (as evidenced by Fig. (1a)), but was adverse to particle secretion. Conversely, the expressed HBsAg could be efficiently secreted, either in the form of $22 \mathrm{~nm}$ subviral particles or HDV VLPs. The enhanced HBsAg expression at lower Bac-GD dosage (e.g. MOT 2) consequently elevated the overall $\mathrm{HBsAg}$ and LHDAg secretion.

Although uniform VLP size distribution is reported for many viruses $[30,31]$, some exceptions exist. For instance, co-expression of multiple infectious bursal disease virus (IBDV) structural proteins in insect cells gives rise to the emergence of $60 \mathrm{~nm}$ IBDV-like VLP and $20 \mathrm{~nm}$ subviral particles [32]. The infection of HBV, a virus closely pertinent to HDV, produces the complete Dane particles $(\approx 42 \mathrm{~nm}$, buoyant density $\approx 1.22 \mathrm{~g} / \mathrm{cm}^{3}$ ) and two distinct forms of subviral particles consisting of HBsAg: pleomorphic, spherical subviral particles that measure $\approx 22 \mathrm{~nm}$ in diameter (buoyant density $\approx 1.18 \mathrm{~g} / \mathrm{cm}^{3}$ ) and tubular particles of various length (diameter $\approx 20 \mathrm{~nm}$ ). Since the secretion of HDV VLP requires the interactions between L-HDAg and HBsAg, budding through Golgi complex and acquiring of viral envelopes [33], the relative concentrations of L-HDAg and HBsAg localized in the Golgi complex must play a pivotal role in the particle formation and secretion. Decreasing the L-HDAg dosage from MOT 20 to 2 was expected to increase the local concentration of HBsAg, thus leading to the secretion of more $22 \mathrm{~nm}$ HBsAg particles (as judged by the increased percentage of HBsAg particles from $5.8 \%$ to $21.2 \%$, Table 1) and the incorporation of more HBsAg into the HDV VLP (as evidenced by the decreased D/B ratio in the HDV VLP from 1.26 to 0.72 ). The enhanced packaging of HBsAg into the HDV VLP, and the increased association with lipids, led to a decrease in the particle density distribution, as well as the reduction in the average particle size and the diminishing of $>40 \mathrm{~nm}$ HDV VLP.

Although HDV VLPs have been produced using recombinant yeasts [10], mammalian cells co-transfected with plasmids [29], or mammalian cells co-transduced with bacu-

Table 1. Summary of the VLP Properties at Different MOT

\begin{tabular}{|c|c|c|c|c|c|}
\hline Bac-GD MOT & $\operatorname{HBsAg}^{1}$ (ng) & L-HDAg² (ng) & HBsAg Particle ${ }^{3}(\%)$ & HDV VLP ${ }^{4}(\%)$ & D/B Ratio ${ }^{5}$ \\
\hline 20 & 31.3 & 39.4 & 5.8 & 94.2 & 1.26 \\
\hline 5 & 62.5 & 53.9 & 10.1 & 89.9 & 0.86 \\
\hline 2 & 83.1 & 59.9 & 21.2 & 78.8 & 0.72 \\
\hline
\end{tabular}

HBsAg represents the total HBsAg secreted to the extracellular medium from $10^{7}$ cells for the synthesis of HBsAg particles (22 nm) and HDV VLP.

${ }^{2} \mathrm{~L}-\mathrm{HDAg}$ represents the total L-HDAg secreted to the extracellular medium from $10^{7}$ cells for the synthesis of HDV VLP.

${ }^{3} \mathrm{HBs}$ Ag particles represents the subviral particles self-assembled from HBsAg alone, with a diameter range of 20-25 nm.

${ }^{4} \mathrm{HDV}$ VLP represents the empty particles consisting of HBsAg and L-HDAg with a diameter greater than $25 \mathrm{~nm}$.

${ }^{5} \mathrm{D} / \mathrm{B}$ ratio represents the weight ratio of L-HDAg/HBsAg in the HDV VLP. 
loviruses $[22,23]$, the diameters of the resultant particles generally fall in the range between $22 \mathrm{~nm}$ (HBsAg subviral particles) and $37 \mathrm{~nm}$ (HDV VLP). To our best knowledge, this is the first study reporting the production of particles greater than $50 \mathrm{~nm}$ in diameter by the co-expression of HBsAg and L-HDAg. The discrepancy between our discovery and previous findings probably arose from the exceedingly high level expression of L-HDAg in this study, particularly at Bac-GD MOT 20. It was reported that the ratio of LHDAg to HBsAg in purified HDV VLPs is approximately 0.12 (from yeast) or 0.1 (from transfected Huh-7 cells) [10] and the 20-25 nm HBsAg particles accounted for $>40 \%$ of total particle populations [10], suggesting that HBsAg expression was in excess. Similarly, we previously cotransduced the cells with Bac-GS2 and Bac-GD at a volumetric ratio of 4 (no MOT was determined in that study), which resulted in the production of particles predominantly resembling the $37 \mathrm{~nm}$ HDV virion. These data concurred with our data obtained at MOT 2. In this study, adjusting the Bac-GD MOT at 20 while fixing the Bac-GS2 MOT at 10 resulted in significantly high L-HDAg expression level that was unparalleled, thus enabling the discovery of the distinct particles.

\section{CONCLUSIONS}

In summary, this study demonstrated that manipulating the relative expression of HBsAg and L-HDAg profoundly influenced the protein secretion, particle density, size distribution, and VLP composition. Strikingly, high level expression of L-HDAg relative to HBsAg (e.g. in event of MOT $20)$ led to the emergence of unusual oversize particles. Albeit the obscure biological roles of these oversize particles, this study highlights the significance in manipulating the recombinant baculovirus dosage to control the VLP composition. Understanding the dependence of VLP properties on the baculovirus dosage is critical for future VLP production and purification, especially when the VLP is intended to be used for immunological study or vaccination.

\section{ACKNOWLEDGEMENTS}

The authors wish to thank Prof. P.J. Chen for providing the purified S-HDAg and Prof. S.C. Lee for providing antibody A10F1. The authors also gratefully acknowledge the financial support from the National Health Research Institutes (NHRI-EX96-9412EI) and VTY Joint Research Program, Tsou's Foundation (VHGUST95-P6-17), Taiwan.

\section{REFERENCES}

[1] Rizzetto M, Canese MG, Arico S, Crivelli O, Trepo C, Bonino F, Verme G. Immunofluorescence detection of new antigen-antibody system (delta/anti-delta) associated to hepatitis B virus in liver and in serum of HBsAg carriers. Gut 1977; 18:997-1003.

[2] He L-F, Ford E, Purcell RH, London WT, Philips J, Gerin JL. The size of hepatitis delta agent. J Med Virol 1989; 27:31-3.

[3] Chang FL, Chen PJ, Tu SJ, Wang CJ, Chen DS. The large form of hepatitis antigen is crucial for assembly of hepatitis virus. Proc Natl Acad Sci USA 1991; 88:8490-4.

[4] Taylor J, Chao M, Hsieh SY, Ryu WS. The roles of the deltaantigen in the structure and replication of hepatitis-delta virus. J Hepatol 1991; 13:S119-S20.

[5] Schiller JT, Lowy DR. Papillomavirus-like particle based vaccines: cervical cancer and beyond. Expert Opin Biol Ther 2001; 1:571-81.
[6] Roy P. Genetically engineered particulate virus-like structures and their use as vaccine delivery systems. Intervirology 1996; 39:62-71.

[7] Lenz P, Day PM, Pang YYS, Frye SA, Jensen PN, Lowy DR, Schiller JT. Papillomavirus-like particles induce acute activation of dendritic cells. J Immunol 2001; 166:5346-55.

[8] Ryu WS, Bayer M, Taylor J. Assembly of hepatitis delta virus particles. J Virol 1992; 66:2310-5.

[9] Wang CJ, Chen PJ, Wu JC, Patel D, Chen DS. Small-form hepatitis B surface antigen is sufficient to help in the assembly of hepatitis delta virus-like particles. J Virol 1991; 65:6630-6.

[10] Wu HL, Chen PJ, Mu JJ, et al. Assembly of hepatitis delta viruslike empty particles in yeast. Virology 1997; 236:374-81.

[11] Kost TA, Condreay JP, Jarvis DL. Baculovirus as versatile vectors for protein expression in insect and mammalian cells. Nat Biotechnol 2005; 23:567-75.

[12] $\mathrm{Hu}$ Y-C. Baculovirus as a highly efficient expression vector in insect and mammalian cells. Acta Pharmacol Sin 2005; 26:405-16.

[13] $\mathrm{Hu}$ Y-C. Baculovirus vectors for gene therapy. Adv Virus Res 2006; 68:287-320.

[14] Condreay JP, Ames RS, Hassan NJ, et al. Baculoviruses and mammalian cell-based assays for drug screening. Adv Virus Res 2006; 68:255-86.

[15] Makela AR, Oker-Blom C. Baculovirus display: A multifunctional technology for gene delivery and eukaryotic library development. Adv Virus Res 2006; 68:91-112.

[16] Wang C-Y, Li F, Yang Y, Guo H-Y, Wu C-X, Wang S. Recombinant baculovirus containing the Diphtheria toxin A gene for malignant glioma therapy. Cancer Res 2006; 66:5798-806.

[17] Yang D-G, Chung Y-C, Lai Y-K, Lai C-W, Liu H-J, Hu Y-C. Avian influenza virus hemagglutinin display on baculovirus envelope: Cytoplasmic domain affects virus properties and vaccine potential. Mol Ther 2007; 15:989-96.

[18] Sung L-Y, Lo W-H, Chiu H-Y, et al. Modulation of chondrocyte phenotype via baculovirus-mediated growth factor expression. Biomaterials 2007; 28:3437-47.

[19] Ho Y-C, Lee H-P, Hwang S-M, et al. Baculovirus transduction of human mesenchymal stem cell-derived progenitor cells: variation of transgene expression with cellular differentiation states. Gene Ther 2006; 13:1471-9.

[20] Zeng J, Du J, Zhao Y, Palanisamy N, Wang S. Baculoviral vectormediated transient and stable transgene expression in human embryonic stem cells. Stem Cells 2007; 25:1055-61.

[21] Chuang C-K, Sung L-Y, Hwang S-M, Lo W-H, Chen H-C, Hu YC. Baculovirus as a new gene delivery vector for stem cells engineering and bone tissue engineering. Gene Ther 2007; Accepted.

[22] Wang K-C, Wu J-C, Chung Y-C, Ho Y-C, Chang MD, Hu Y-C. Baculovirus as a highly efficient gene delivery vector for the expression of hepatitis delta virus antigens in mammalian cells. Biotechnol Bioeng 2005; 89:464-73.

[23] Chen Y-H, Wu J-C, Wang K-C, et al. Baculovirus-mediated production of HDV-like particles in BHK cells using a novel oscillating bioreactor. J Biotechnol 2005; 118:135-47.

[24] Hu Y-C, Bentley WE. Effect of MOI ratio on the composition and yield of chimeric infectious bursal disease virus-like particles by baculovirus co-infection: Deterministic predictions and experimental results. Biotechnol Bioeng 2001; 75:104-19.

[25] O'Reilly D, Miller L, Luckow V. Baculovirus expression vectors: a laboratory manual. New York: W.H. Freeman and Co, 1992.

[26] Chan Z-R, Lai C-W, Lee H-P, Chen H-C, Hu Y-C. Determination of the baculovirus transducing titer in mammalian cells. Biotechnol Bioeng 2006; 93:564-71.

[27] Lee H-P, Chen Y-L, Shen H-C, Lo W-H, Ho Y-C, Hu Y-C. Baculovirus transduction of rat articular chondrocytes: Roles of cell cycle. J Gene Med 2007; 9:33-43.

[28] Hsu SC, Syu WJ, Ting LT, Wu JC. Immunohistochemical differentiation of hepatitis D virus genotypes. Hepatology 2000; 32:1111-6.

[29] Wu JC, Chen PJ, Kuo MY, Lee SD, Chen DS, Ting LP. Production of hepatitis delta virus and suppression of helper hepatitis B virus in a human hepatoma cell line. J Virol 1991; 65:1099-104.

[30] Urakawa T, Ferguson M, Minor PD, et al. Synthesis of immunogenic, but non-infectious poliovirus particles in insect cells by a baculovirus expression vector. J Gen Virol 1989; 70:1453-63. 
[31] Volpers C, Schirmacher P, Streeck RE, Sapp M. Assembly of the major and the minor capsid protein of human papillomavirus type 33 into virus-like particles and tubular structures in insect cells. Virology 1994; 200:504-12.

[32] Hu Y-C, Bentley WE, Edwards GH, Vakharia VN. Chimeric infectious bursal disease virus-like particles expressed in insect cells and purified by immobilized metal affinity chromatography. Biotechnol Bioeng 1999; 63:721-9.

[33] Satoh O, Umeda M, Imai H, Tunoo H, Inoue K. Lipid composition of hepatitis B virus surface antigen particles and the particleproducing human hepatoma cell lines. J Lipid Res 1990; 31:1293300 . 